\title{
Implementation of a Patient Blood Management Program in a Large, Diverse Multi-Hospital System
}

Sumita Markan-Aurora, MD, Rachel Miller, MSN, RN, Audrey A. Gronemeyer, MPH, Jennifer S. Kempf, MS, and Bruce L. Hall, MD, PhD, MBA

\section{ABSTRACT}

Background: There is limited literature relating to patient blood management (PBM) programs in large multi-hospital systems or addressing challenges of implementation across diverse systems comprised of community and academic hospitals.

Objective: To establish a PBM program to improve utilization of blood transfusion units at a multi-hospital system in the Midwest (BJC HealthCare).

Methods: High-impact strategies in establishing the PBM program included formation of Clinical Expert Councils (CECS) of providers, establishment of consensus utilization guidelines, and development of a robust reporting tool. CECs enabled collaboration and facilitated standardization across a complex system of academic, private practice, and tertiary facilities with a diverse community of medical providers. Consensus guidelines and the PBM reporting tool were key to creating meaningful reports to drive provider practice change.
Results: Over the 5 years following implementation of the PBM program, there has been a steady decrease in red blood cell (RBC) utilization. Noticeable changes have taken place at individual hospitals in the system, including reductions in transfusions falling outside guideline parameters from 300 per quarter to less than 8 per quarter at 1 of our community hospitals. No negative impact on patient care has been identified.

Conclusion: In response to current transfusion guidelines and the need for optimizing stewardship of blood product resources, this hospital system successfully implemented a robust PBM program that engaged academic and non-academic community providers and decreased utilization of blood transfusion resources in line with consensus guidelines.

Keywords: quality improvement; RBC transfusion; transfusion practices; provider practice change; utilization trends.

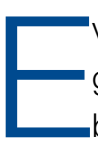
vidence from clinical trials and published clinical guidelines support the adoption of a restrictive blood transfusion approach in hospitalized, stable patients as best practice..$^{1-5}$ As such, the development and implementation of patient blood management (PBM) programs has become an increasingly important process improvement for reducing variability in transfusion practices and clinical outcomes.

As recently as 2013, BJC HealthCare, a multihospital system in the Midwest, had no standardized, system-wide blood management program, and transfusion practices varied widely across providers and between individual hospitals based on size, patient population, and resources. The system consisted of 13 hospitals, ranging from large tertiary to smaller commu- nity and academic hospitals. Although adults constituted the vast majority of the patient population, the hospital system also included a pediatric specialty hospital, St. Louis Children's Hospital. In addition, some sites were staffed by private practice providers and others by university-based providers, including blood bank medical directors. Due to the diversity of settings and populations, efforts to align transfusion and other practices often faced multiple challenges. However, improving the management of blood transfusions was identified as a key resource stewardship priority in 2013, and implementation of a system-wide program began after extensive discussions and consensus approval by senior

From BJC HealthCare, St. Louis, MO. 


\section{Patient Blood Management}

hospital system and medical leadership. The primary aim of the program was to optimize overall blood product resource stewardship. Specifically, we sought to control or reduce costs per patient-care episode using strategies that would not negatively impact patient care and could potentially even improve patient outcomes (eg, by avoiding unnecessary transfusions and their attendant risks).

There is a plethora of literature related to the implemention of PBM programs in individual hospitals, ${ }^{6-18}$ but few reports specifically relate to large multi-hospital health systems, ${ }^{19-21}$ or directly address the unique challenges of implementation across a diverse system of community and academic hospitals and providers. ${ }^{19}$ Here, we discuss our experience with establishing a PBM program in a large, diverse, multi-hospital health system, provide examples of innovative strategies, and address challenges faced and lessons learned. Future endeavors of the PBM program at BJC HealthCare are also described.

\section{Setting}

BJC HealthCare is one of the largest nonprofit health care organizations in the United States, delivering services to the greater St. Louis, southern Illinois, and mid-Missouri regions, and addressing the health care needs of urban, suburban, and rural communities. As of 2018, the system included 15 hospitals and multiple community health locations comprising more than 3400 staffed beds, 31,500 employees, and 4300 physicians with privileges. The system annually has more than 151,000 hospital admissions, 81,000 outpatient surgery visits, and 537,000 emergency department visits. In addition to inpatient and outpatient care, services include primary care, community health and wellness, workplace health, home health, community mental health, rehabilitation, long-term care, and hospice. As a nonprofit system, BJC is the largest provider of charity care, unreimbursed care, and community benefit in Missouri, highlighting the fact that resource stewardship is a critical issue across the entire system and the communities served. 22

\section{PBM Project}

Preparation for large-scale change across several hospitals began with creating a framework for the initiative, which consisted of a "burning platform," a guiding vision, and a coalition. The burning platform identifies the importance and urgency of a change and helps to establish commitment. Between 2012 and 2014, the American Association of Blood Banks (AABB) released new evidence-based guidelines and recommendations calling for more restrictive transfusion practices pertaining to red blood cells (RBCs; ie, a hemoglobin threshold of 7 to $8 \mathrm{~g} / \mathrm{dL}$ ) in both inpatient and outpatient care. ${ }^{2}$ In addition, use of single-unit transfusions was recognized as best practice by the AABB in the Choosing Wisely campaign. ${ }^{23}$ Historically, adult patients requiring transfusions were given 2 units in succession. The new recommendations provided a strong basis for changing transfusion practices at BJC. It was believed that aligning transfusion practices with the new guidelines was consistent with the mission and vision of the work: that these changes could lead to optimization of resources, cost control, reductions in unnecessary blood transfusions, and potentially improved care (eg, fewer transfusionrelated complications). We used the national guidelines to initiate discussions and to identify clinical conditions and associated laboratory parameters for transfusion therapy.

Once this burning platform was established, a team comprised of physicians, blood bank experts, quality consultants, data analysts, and supply managers, referred to as the Outcomes Team, was formed to lead the change efforts across the system. Initial projects for the team included developing system-wide consensus-based transfusion guidelines, providing education to providers on the new evidence in transfusion practice, and sharing BJC-specific historical utilization data. The guiding principle for the group was that "blood is a valuable resource, but not without risk, and less is more." In order to disseminate the vision of the initiative across the system, campaign signs with the slogans " 7 is the new 10" (referring to the $\mathrm{g} / \mathrm{dL}$ transfusion threshold) and " 1 is the new 2" (referring to the new practice of the preferential transfusion of single units rather than 2 at a time) were displayed in system hospitals.

Last, a guiding coalition of system leaders was needed to help push the initiative forward and sustain the program once fully implemented. Thus, a multidisciplinary PBM Clinical Expert Counciel (CEC) was formed to assist with implementation and maintenance of the program. 


\section{Role of PBM Clinical Expert Council}

The PBM CEC was designed to improve overall physician and expert engagement and provide a forum where stakeholders from across the system could participate to voice their expert opinion. CECs (which BJC formed in other clinical areas as well) are multidisciplinary teams consisting of clinical, administrative, and technical staff. The open, multidisciplinary structure of the councils allows for collaboration that promotes change across a complex multi-hospital system. Each hospital is represented by key physicians and technical leaders, opening opportunity for both horizontal and vertical partnership.

As part of the overall physician engagement strategy, the PBM CEC was launched across BJC in November 2013 as a decision-making body for gaining system consensus on matters relating to blood management. The initial goals for the PBM CEC were to share information and educate providers and others on the latest evidence, to subsequently debate and develop consensus for guidelines to be applied across BJC, and to identify and adopt gold standard practices to drive and sustain compliance across the system. More specifically, we wanted to focus on how to avoid unnecessary blood transfusions known to be associated with increased risk for adverse reactions, other morbidity, mortality, and longer length of stay. Council members met quarterly to address 6 key drivers: patient safety, informatics and data, quality improvement, efficiencies and workflows, education and competency, and communication and engagement. Members then voted to approve guidelines, policies, and procedures. The group continues to assist in updating and standardizing guidelines and providing input on improving the functionality of the PBM reporting tool.

\section{Development of the PBM Reporting Tool}

Providing and sharing data on blood utilization and practices with the CEC and hospital leaders was imperative to driving change. The Outcomes Team deliberated on how best to generate and provide such information, conducting comparisons between selected vendor-based tools and potential internal BJC solutions. After investigation, BJC leadership approved the development of an in-house PBM dashboard tool using Tableau Desktop
(Tableau Software, Inc.). The tool consists of an executive page with 5 additional tabs for navigating to the appropriate information (Figure 1 and Figure 2); data within the tool are organized by facility, service, provider, ICD diagnosis, transfusion indication, and the Clinical Classifications Software category, as defined by the Agency for Healthcare Research and Quality.

The PBM reporting tool was launched on December 31, 2014. The next priority after the launch was to validate the tool's blood utilization data and implement enhancements to make the tool more effective for users. A super-user group consisting of blood bank supervisors and managers was established. The goals of the user group were to preview any enhancements before presenting the tool to the larger CEC, test and validate data once new information was added, and share and prioritize future enhancements. User group meetings were held monthly to share best practices and discuss individual facilities' blood utilization data. In addition, each facility's representative(s) shared how they were driving changes in provider practice and discussed challenges specific to their facility. Enhancements suggested through the user group included: incorporation of additional lab values into the tool to correspond with other blood products (eg, fibrinogen, hematocrit, international normalized ratio, and platelet count), addition of the specific location where the blood product was administered, and standard naming conventions of locations to allow comparisons across facilities (eg, Emergency Department instead of $\mathrm{ED}$, ER, or EU).

All hospital users were given access to a test version of the reporting tool where they could review enhancements, identify what worked well and what could be done better, and suggest corrections. As changes were made to the hospital lab systems, a sample of data was reviewed and validated with affected facilities to confirm the continued accuracy of the data. To ensure its practicality to users, the tool continues to be improved upon with input from council stakeholders and subject-matter experts.

\section{Measurements}

To monitor blood utilization across the health system, we tracked the total RBC units administered by hospital, service, and provider and also tracked pre- and post-transfusion hemoglobin values. 


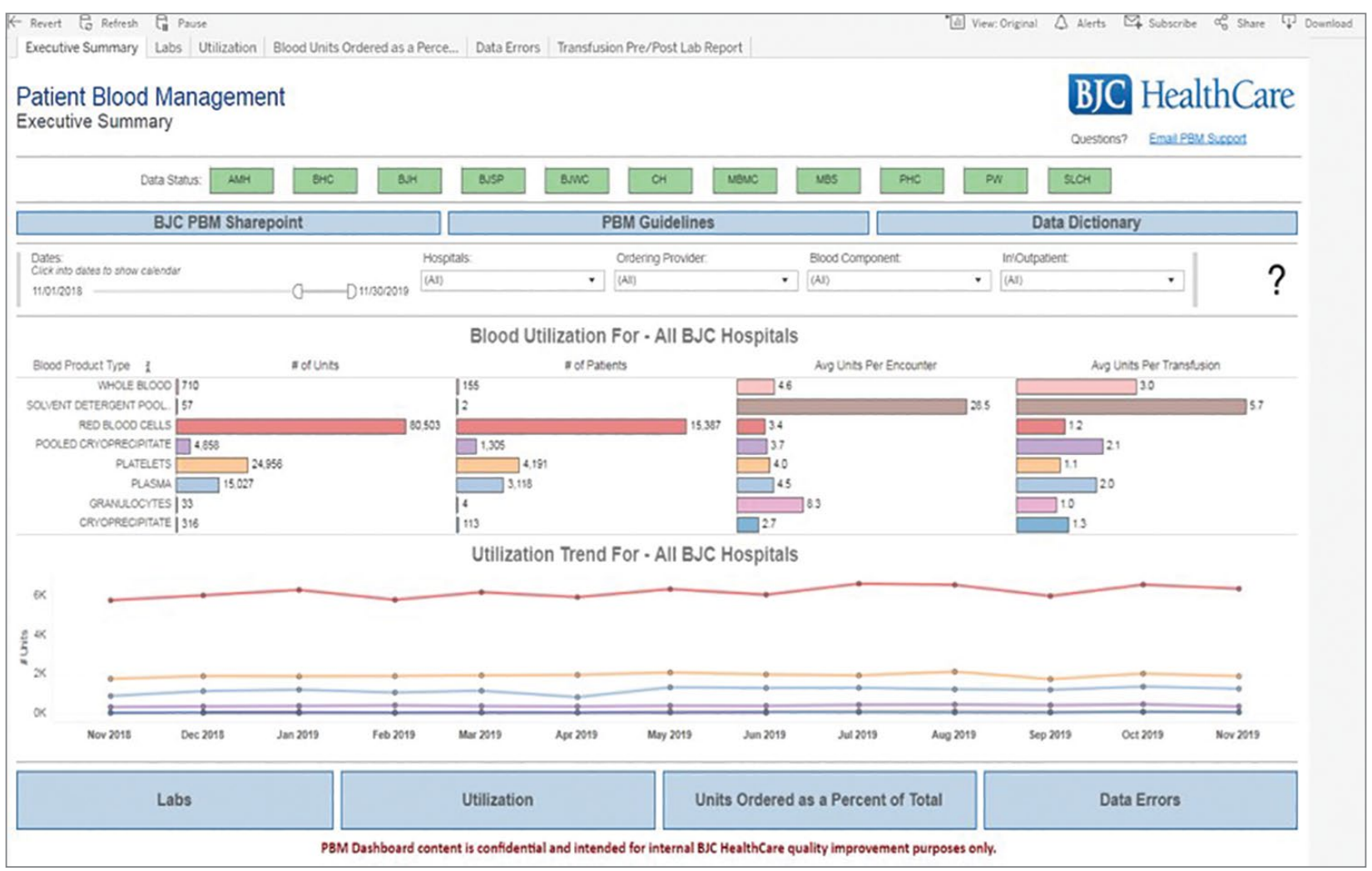

Figure 1. Executive page of the Patient Blood Management dashboard (November 2018 to November 2019).

\section{Results}

Overall, the system has seen a steady decrease in RBC utilization over the 5 years since the PBM program was implemented (Table), with an absolute decrease of 3998 RBC units utilized per year from 2013 to 2018 . From this assessment, focus efforts are being identified and future work will incorporate targeted metrics for those areas with higher utilization of RBCs. More importantly, from 2014 through 2017, there was a consistent decreasing trend in the number of transfusion-related safety events (302 to 185, respectively). However, there was a slight increase in reported events from 2018 to 2019 (188 and 266, respectively).

In addition to system-wide improvement, noticeable changes have taken place at individual hospitals in the BJC system. For example, Boone Hospital Center in Columbia, Missouri, began critically reviewing all RBC transfusions starting in 2015 and, to raise awareness, communicating with any provider who transfused a patient outside of transfusion guidelines. Since then, Boone Hospital has seen a dramatic reduction in transfusions considered noncom- pliant (ie, falling outside guideline parameters), from 300 transfusions per quarter, down to less than 8 per quarter. St. Louis Children's Hospital also began reviewing blood products utilized by providers that fell outside of the standardized guidelines. At this hospital, physician champions discuss any outliers with the providers involved and use multiple methods for disseminating information to providers, including grand rounds, faculty meetings, and new resident orientations.

Another success has been the partnership between Barnes Jewish St. Peters and Progress West Hospitals in providing PBM education. Their joint effort resulted in implementation of education modules in BJC's internal learning system, and has provided PBM-related education to more than 367 nurses, blood bank staff, and physicians.

\section{Challenges and Lessons Learned}

Implementation of the PBM program was generally successful, but it was not without challenges. One of the biggest challenges was addressing the variation in care and 


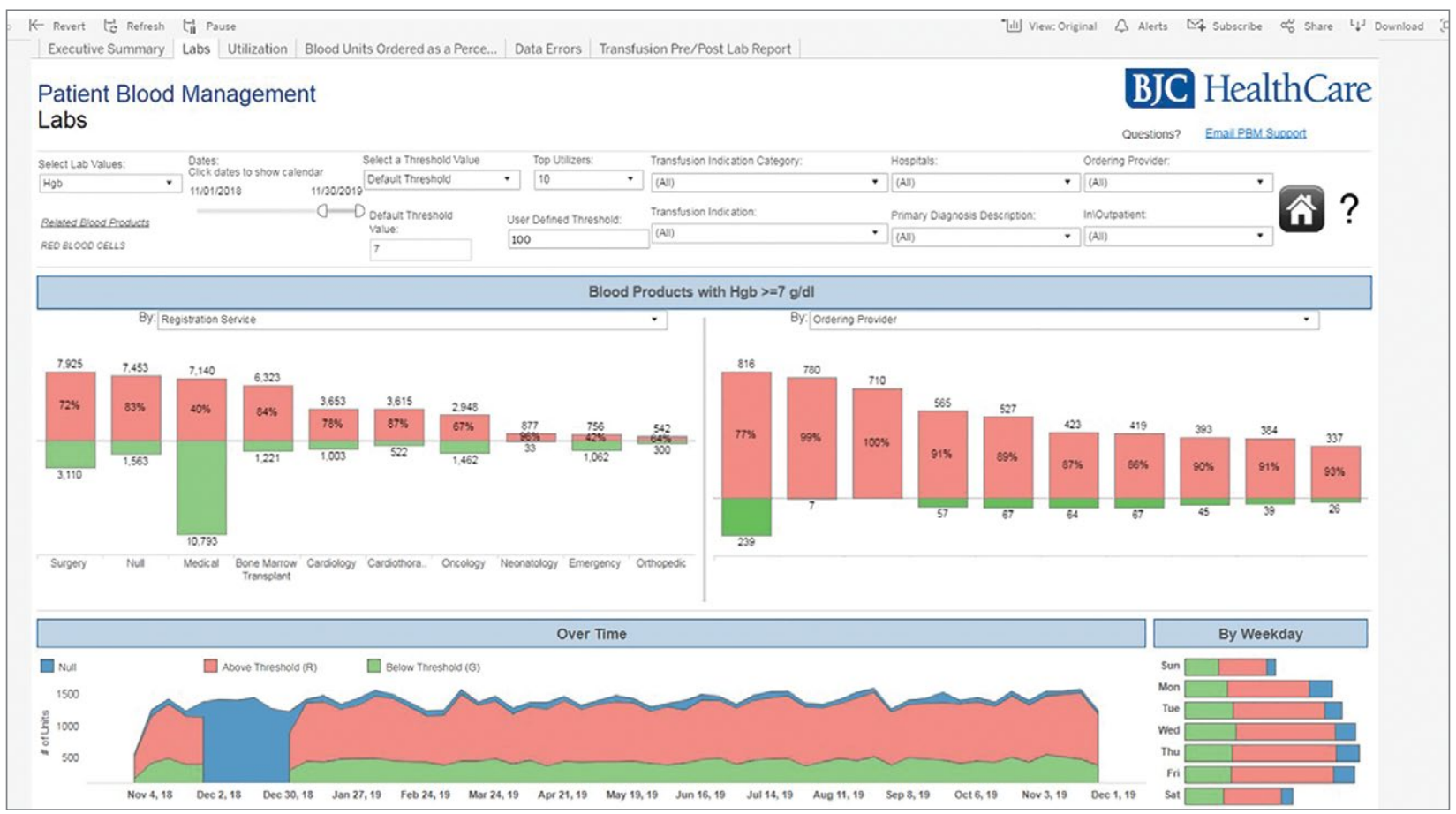

Figure 2. Labs tab of Patient Blood Management dashboard-transfusions with hemoglobin $<7 \mathrm{~g} / \mathrm{dL}$.

practices across the hospital enterprise. Due to the varying sizes and service goals of individual hospitals, lack of standardization was a significant barrier to change. Gaining trust and buy-in was imperative to increasing compliance with new transfusion policies. The primary concern was finding a balance between respecting physician autonomy and emphasizing and aligning practices with new evidence in the literature. Thus, understanding and applying principles of thoughtful change management was imperative to advancing the framework of the PBM program. The CEC venue enabled collaboration among hospitals and staff and was ultimately used to facilitate the necessary standardization process. To gain the trust of hospital and medical staff, the Outcomes Team conducted several site visits, enabling face-to-face interaction with frontline staff and operational leaders. Moreover, the team's emphasis on the use of the latest evidence-based guidelines in discussions with hospital and medical staff underscored the need for change.

Frank et a ${ }^{19}$ describes using an approach similar to our Outcomes Team at the Johns Hopkins Health System. A designated multidisciplinary quality improvement team, referred to as the "clinical community," worked on implementing best practices for blood management across a system of 5 hospitals. The authors reported similar results, with an overall decrease in number of units transfused, as well as substantial cost savings. ${ }^{19}$ Our project, along with the project implemented by Frank et al, shows how a "consensus-community" approach, involving stakeholders and various experts across the system, can be be used to align practices among multiple hospitals.

Development of a robust PBM reporting tool was key to creating meaningful monthly reports and driving provider practice change. However, this did require several training sessions, site visits, and computer-based training. Members of the Outcomes Team engaged in oneon-one sessions with tool users as a way of addressing specific areas of concern raised by staff at individual blood banks, and also took part in system-wide initiatives. The team also attended blood bank staff meetings and hospital transfusion committee meetings to educate staff on the evidence and initiative, provide demos of the reporting tool, and allow for a more robust discussion of how the data could be used and shared with other 
Table. Red Blood Cell Units Dispensed From BJC Blood Banks

\begin{tabular}{lccc}
\hline Year & Total RBC Units & $\begin{array}{c}\text { Average RBC Units } \\
\text { per Transfusion }\end{array}$ & $\begin{array}{c}\text { RBC Units } \\
\text { per 1000 CMI APD* }\end{array}$ \\
\hline 2013 & 77,229 & No data & 3101 \\
\hline 2014 & 75,914 & 1.3 & 2875 \\
\hline 2015 & 73,003 & 1.3 & 2578 \\
\hline 2016 & 73,667 & 1.3 & 2409 \\
\hline 2017 & 73,909 & 1.3 & 2316 \\
\hline 2018 & 73,231 & 1.2 & 2241 \\
\hline 2019 annualized & 75,178 & 1.2 & 2249
\end{tabular}

APD, adjusted patient days; CMI, case mix index; RBC, red blood cell.

*Adjusted by volume (adjusted patient days) and patient acuity (case mix index).

departments. These sessions provided opportunities to identify and prioritize future enhancements, as well as opportunities for continued education and discussion at hospitals, which were critical to ongoing improvement of the reporting tool.

\section{Conclusion and Future Directions}

Blood products remain extremely valuable and scarce resources, and all health care professionals must work to prevent unnecessary transfusions and improve clinical outcomes by adhering to the latest evidence-based guidelines. In response to current transfusion guidelines and the need to optimize blood product resources, our system successfully implemented a robust PBM program that engaged both academic and non-academic providers and communities. Several elements of the program helped us overcome the challenges relating to standardization of transfusion practices: consensus-based development of guidelines using the latest scientific evidence; formation and utilization of the CEC venue to gain system-wide consensus around both guidelines and approaches to change; development of a trustworthy and accessible PBM reporting tool (as well as continuing education sessions to improve adoption and utilization of the tool); and ongoing multidisciplinary discussions and support of thoughtful change and sustaining activities. We have seen a system-wide decrease in the number of RBC units transfused (absolute and per case mixadjusted patient day) since implementing the PBM program, and in the following years have noted a trending decrease in transfusion-related safety events. Although there was a slight increase in reported safety events from 2018 to 2019, this was likely due to the systematic implementation of a new electronic medical record system and improved reporting infrastructure.

Upcoming phases of our system-wide PBM program will include looking at opportunities to improve blood utilization in other specific clinical areas. For example, we have begun discussions with hematology and oncology experts across the system to expand their patient population data within the PBM reporting tool, and to identify areas of opportunity for provider practice change within their specialty. We are also reviewing cardiothoracic surgery transfusion data to identify opportunities for reducing blood utilization in specific clinical scenarios. In addition, we are working to incorporate our 2 newest hospital system members (Memorial Hospital East and Memorial Hospital Belleville) into the PBM program. In collaboration with perioperative leaders across the system, the surgical blood ordering process is being reviewed. The goal of this effort is to reduce blood products ordered in preparation for surgical procedures. We are also currently investigating whether an impact on safety events (ie, reduction in transfusion reactions) can yet be detected. Last, our health care system recently launched a system-wide electronic medical record, and we are eager to see how this will provide us with new methods to monitor and analyze blood administration and utilization data. We look forward to reporting on the expansion of our program and on any clinical outcome 
improvements gained through avoidance of unnecessary transfusions.

Acknowledgment: The authors thank the leadership within the Center for Clinical Excellence and Supply Chain at BJC HealthCare for their support of this manuscript, as well as all system participants who have contributed to these efforts, especially Mohammad Agha, MD, MHA, current physician leader of the PBM CEC, for his thoughtful edits of this manuscript.

Corresponding author: Audrey A. Gronemeyer, MPH, Center for Clinical Excellence, BJC HealthCare, 8300 Eager Road, Suite 400A, St. Louis, MO 63144; audrey.gronemeyer@bjc.org.

Financial disclosures: None.

\section{References}

1. Carson JL, Grossman BJ, Kleinman S, et al. Red blood cell transfusion: A clinical practice guideline from the $\mathrm{AABB}^{*}$. Ann Intern Med. 2012;157:49-58.

2. Goodnough LT, Levy JH, Murphy MF. Concepts of blood transfusion in adults. Lancet. 2013;381:1845-1854.

3. Hébert PC, Carson JL. Transfusion threshold of $7 \mathrm{~g}$ per deciliterThe new normal. N Engl J Med. 2014;371:1459-1461.

4. Gani F, Cerullo M, Ejaz A, et al. Implementation of a blood management program at a tertiary care hospital: Effect on transfusion practices and clinical outcomes among patients undergoing surgery. Ann Surg. 2019;269:1073-1079.

5. Podlasek SJ, Thakkar RN, Rotello LC, et al. Implementing a "why give 2 when 1 will do?" Choosing Wisely campaign. Transfusion. 2016;56:2164.

6. Boral LI, Bernard A, Hjorth T, et al. How do I implement a more restrictive transfusion trigger of hemoglobin level of $7 \mathrm{~g} / \mathrm{dL}$ at my hospital? Transfusion. 2015;55:937-945.

7. Geissler RG, Kosters C, Franz D, et al. Utilization of blood components in trauma surgery: A single-center, retrospective analysis before and after the implementation of an educative PBM initiative. Transfuse Med Hemother. 2015;42:83-89.

8. Goel R, Cushing MM, Tobian AA. Pediatric patient blood management programs: Not just transfusing little adults. Transfus Med Rev. 2016;30:235-241.

9. Gupta PB, DeMario VM, Amin RM, et al. Patient blood management program improves blood use and clinical outcomes in orthopedic surgery. Anesthesiology. 2018;129;1082-1091.
10. Leahy MF, Roberts $H$, Mukhtar SA, et al. A pragmatic approach to embedding patient blood management in a tertiary hospital. Transfusion. 2014;54:1133-1145.

11. Leahy MF, Hofmann A, Towler S, et al. Improved outcomes and reduced costs associated with a health-system-wide patient blood management program: A retrospective observational study in four major adult tertiary-care hospitals. Transfusion. 2017; 57:1347-1358.

12. Meybohm P, Herrmann E, Steinbicker AU, et al. Patient blood management is associated with a substantial reduction of red blood cell utilization and safe for patient's outcome: A prospective, multicenter cohort study with a noninferiority design. Ann Surg. 2016;264:203-211.

13. Morgan PN, Coleman PL, Martinez-Garduno CM, et al. Implementation of a patient blood management program in an Australian private hospital orthopedic unit. $J$ Blood Med. 2018;9;83-90.

14. Norgaard A, Stensballe J, de Lichtenberg TH, et al. Three-year follow-up of implementation of evidence-based transfusion practice in a tertiary hospital. Vox Sang. 2017;112:229-239.

15. Meuller MM, Van Remoortel H, Meybohm P, et al. Patient blood management: Recommendations from the 2018 Frankfurt Consensus Conference. JAMA. 2019;321:983-997.

16. Oliver JC, Griffin RL, Hannon T, Marques MB. The success of our patient blood management program depended on an institution-wide change in transfusion practices. Transfusion. 2014; 54:2617-2624.

17. Thakkar RN, Lee KH, Ness PM, et al. Relative impact of a patient blood management program on utilization of all three major blood components. Transfusion. 2016;56:2212-2220.

18. Yang WW, Thakkar RN, Gehrie EA, et al. Single-unit transfusions and hemoglobin trigger: relative impact on red cell utilization. Transfusion. 2017;57:1163-1170.

19. Frank SM, Thakkar RN, Podlasek SJ, et al. Implementing a health system-wide patient blood management program with a clinical community approach. Anesthesiology. 2017;127;754-764.

20. Verdecchia NM, Wisniewski MK, Waters JH, et al. Changes in blood product utilization in a seven-hospital system after the implementation of a patient blood management program: A 9-year follow-up. Hematology. 2016;21:490-499.

21. Yazer MH, Waters JH. How do I implement a hospital-based blood management program? Transfusion. 2012;52:1640-1645.

22. BJC HealthCare. Facts and Figures.. BJC HealthCare website. www.bjc.org/About-Us/Facts-Figures. Accessed November 18, 2019.

23. Callum JL, Waters $\mathrm{JH}$, Shaz BH, et al. The AABB recommendations for the Choosing Wisely campaign of the American Board of Internal Medicine. Transfusion. 2014;54:2344-2352. 